\title{
Associations of sarcopenia and its defining components with cognitive function in community-dwelling oldest old
}

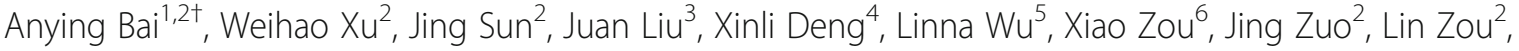 \\ Yunxia Liu ${ }^{2}$, Hengge Xie ${ }^{7}$ Xiaohong Zhang ${ }^{7}$, Li Fan ${ }^{2}$ and Yixin $\mathrm{Hu}^{2^{*+}}$
}

\begin{abstract}
Background: This study aimed to investigate the associations of sarcopenia and its defining components with cognitive function in community-dwelling oldest old (over 80 years old) in China.

Methods: Sarcopenia was diagnosed by the 2019 Asian Working Group for Sarcopenia (AWGS) criteria. Cognitive function was evaluated by the Montreal Cognitive Assessment (MoCA). Logistic and linear regression models were used to explore the associations of sarcopenia and its defining components with risk of mild cognitive impairment $(\mathrm{MCl})$, and performance on multiple cognitive domains among 428 adults aged 80 years and older.

Results: The overall prevalence of sarcopenia was 35.5\%, with $40.34 \%$ for men and $32.14 \%$ for women. The prevalence of $\mathrm{MCl}$ was higher among sarcopenic oldest old than non-sarcopenic oldest old $(28.95 \%$ vs. $17.39 \%, p=$ 0.005). Multivariate logistic regression analyses showed that sarcopenia [odds ratio $(\mathrm{OR})=1.86,95 \%$ confidence interval (CI): 1.04-3.33], low handgrip strength (HS) [OR $=2.33,95 \% \mathrm{Cl}: 1.40-3.87]$ and slow gait speed (GS) [OR= 2.31, 95\% Cl: 1.13-4.72] were significantly and independently associated with risk of $\mathrm{MCl}$. Multivariate linear regression analyses showed that low HS was associated with worse performance in global cognitive function, visuospatial and executive function, naming and delayed recall.

Conclusions: Sarcopenia, low HS and low GS was significantly associated with MCl in community-dwelling oldest old. The associations between sarcopenia and its defining components with different cognitive subdomains could be further explored in the future.
\end{abstract}

Keywords: Sarcopenia, Cognitive function, Oldest old, Muscle strength, Gait speed

\section{Background}

Sarcopenia, the age-related decline in skeletal muscle mass concomitant with decreased muscle function [1], has been formally recognized as a geriatric disease with an ICD-10-CM diagnosis code. In 2018, the European Working Group on Sarcopenia in Older People

\footnotetext{
* Correspondence: chhyxcn@126.com

${ }^{\dagger}$ Anying Bai and Yixin Hu contributed equally to this work.

${ }^{2}$ Geriatric Health Care Department of The Second Medical Center \& National Clinical Research Center for Geriatric Diseases, Chinese PLA General Hospital, Beijing, China

Full list of author information is available at the end of the article
}

(EWGSOP2) updated the operational definition and clinical algorithm of sarcopenia by recommending using low muscle strength and low muscle mass to diagnose sarcopenia. Although the newest 2019 consensus of the Asian Working Group for Sarcopenia (AWGS) also contended that diagnosing sarcopenia required both the measurement of muscle mass, muscle strength and gait speed (GS) [2]. An increasing number of studies have indicated that single or multiple components, like muscle strength, is the key element in sarcopenia [3] in recent years. Growing evidence has shown that greater levels of

(c) The Author(s). 2021 Open Access This article is licensed under a Creative Commons Attribution 4.0 International License, which permits use, sharing, adaptation, distribution and reproduction in any medium or format, as long as you give appropriate credit to the original author(s) and the source, provide a link to the Creative Commons licence, and indicate if changes were made. The images or other third party material in this article are included in the article's Creative Commons licence, unless indicated otherwise in a credit line to the material. If material is not included in the article's Creative Commons licence and your intended use is not permitted by statutory regulation or exceeds the permitted use, you will need to obtain permission directly from the copyright holder. To view a copy of this licence, visit http://creativecommons.org/licenses/by/4.0/. The Creative Commons Public Domain Dedication waiver (http://creativecommons.org/publicdomain/zero/1.0/) applies to the data made available in this article, unless otherwise stated in a credit line to the data. 
handgrip strength (HS) is associated with lower risks of cardiovascular disease, all-cause and cardiovascular mortality, physical function, and frailty [4-6]. Therefore, muscle strength is emphasized as the trigger for further assessment and interventions by the introduction of the "probable sarcopenia" concept in the EWGSOP2 definition [7].

As the most prevalent cause of physical impairment, sarcopenia has repeatedly been associated with adverse outcomes such as frailty, hospitalization and increased mortality [7, 8]. Although some basic science and epidemiological studies have suggested a possible shared pathophysiology related to inflammatory markers and the hormonal pathway between sarcopenia and cognitive impairment $[9,10]$, evidence of this association among different populations remains controversial. The large variability was attributed to the methods of defining sarcopenia (based on single or multiple components), the age scope of the studied population and the usage of different detections for cognitive function. For instance, a prospective follow-up study with 555 older adults aged 85 years at baseline reported that poor HS predicted accelerated dependency in activities of daily living (ADL) and global cognitive decline using Mini-Mental State Examination (MMSE) in oldest old [11]. Studies among a population aged 60 years and over also found that low HS was associated with cognitive decline in information processing speed using measures of Digit Symbol Substitution Test (DSST) [12-14]. By contrast, another study included 14,775 Americans at least 50 years old reported that muscle strength capacity and cognitive function may parallel each other [15]. Results from the Tasmanian Study of Cognition and Gait (TASCOG) showed that decline in GS was associated with impairment in executive function, but not other cognitive domains among participants aged 60-85 years [16], while study on Canadians aged older than 65 years old showed no significant association between slower GS and poorer cognitive function detected by MMSE [17]. Moreover, no association was observed between decreased muscle mass and cognitive dysfunction detected by MMSE and the Peterson criteria after $7 \mathrm{y}$ of follow-up in the EPIDOS-Toulouse group [18]. Although MMSE is the most widely used tool when assessing cognitive impairment, some studies report that the Montreal Cognitive Assessment (MoCA) is superior to the MMSE in differentiating mild cognitive impairment (MCI) patients from a healthy control group $[19,20]$.

Aging is an essential risk factor for sarcopenia and decline of cognitive function [21], and has been associated with a reduced activity tolerance attributed to changes in this skeletal muscle blood flow [22]. MCI is the abnormality of cognitive functions in populations matched for age and education levels, but without loss of functional abilities and skills in everyday social and occupational life [23], and it is associated with increased risk of developing dementia. In 2030, there are predicted to be 16.5 million people age 60 or older with dementia, 6.9 million males and 9.6 million females in China [24]. Identifying risk factors associated with $\mathrm{MCI}$ among the oldest old may help to develop early assessment tools for detection of MCI, enabling multi-domain lifestyle interventions at an early phase of dementia [25].

Previous studies showed that associations between handgrip strength, gait speed and cognition were not consistent among oldest-old individuals born in different decades [26], and the associations between blood pressure and cognition were contradictory among young and middle-aged subjects and oldest old subjects [27]. There is scarcity of information on the relationship between components of sarcopenia and different domains of cognitive function among the oldest old in China [28]. Therefore, in this study, we aimed to investigate the association of sarcopenia defined according to the updated 2019AWGS criteria and each of its defining components with performance of whole and multiple cognitive domains in community-dwelling oldest old (over 80 years old) in China.

\section{Methods \\ Data and study participants}

The present study is a cross-sectional analysis of individuals aged 80 years and older in the first wave of an ongoing longitudinal study, which is a group of elders living in a retirement community in Beijing. This study has been approved by the Research Ethics Committee of Chinese PLA General Hospital (Ethic number: S2018-102-02) and registered in Chinese Clinical Trial Register (ChiCTR1900022576). All methods in this study were performed in accordance with the relevant guidelines and regulations. A total of 665 oldest old who had electronic health records from long-run medical centers were recruited from 2018 to 2019 in one retirement community in Beijing, China. The interviews took place in their homes or in person at the community medical center, and information from their electronic health records were also collected. In the present study, people aged 80 years and over were included, those who revealed a history of Parkinson's disease or cancer, or severely impaired ADL were excluded, and our final analysis included 428 participants with a written signed consent. More details on the inclusion process of studied population were provided in Fig. 1 . All of the interviewers were well trained before the study.

\section{Sarcopenia}

According to the AWGS criteria, sarcopenia was diagnosed if participants had low muscle mass plus low 


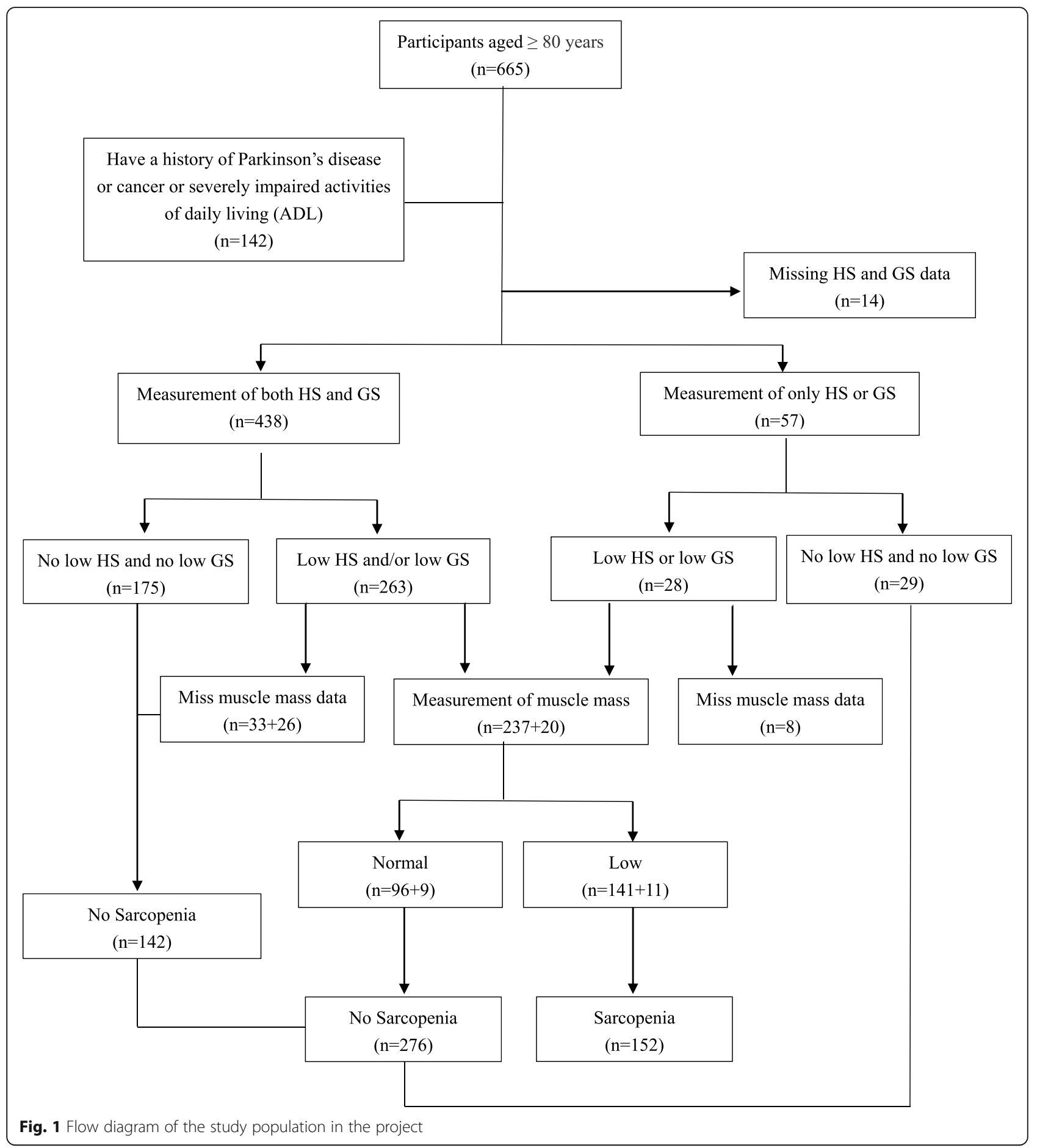

muscle strength or low physical performance [2]. The HS was measured twice for each hand using a digital handgrip dynamometer (JAMAR Co., Ltd., USA), and the maximum value of four tests was analyzed. Low muscle strength was defined as $\mathrm{HS}<28 \mathrm{~kg}$ in men and $<18 \mathrm{~kg}$ in women. Body composition, including fat mass, fat free mass, and skeletal muscle mass, was measured using bioelectrical impedance analysis (BIA,
InBody 270, Biospace Ltd., Seoul, Korea) by qualified research assistants. The appendicular skeletal muscle mass (ASM) was calculated as the sum of lean muscle mass in the arms and legs. According to the recommended method, ${ }^{10}$ skeletal muscle mass index (SMI) was defined as ASM divided by height squared. Low muscle mass was defined as SMI $<7.0 \mathrm{~kg} / \mathrm{m}^{2}$ in men and $<5.7 \mathrm{~kg} / \mathrm{m}^{2}$ in women based on the bioelectrical impedance analysis 
(BIA). Usual GS on a 6-m course was measured objectively, and was used to assess physical performance. Two trials were performed, and the shortest walking time was used to calculate GS and used in the analyses. Slow GS was defined as a gait speed of $\leq 1.0 \mathrm{~m} / \mathrm{s}$ for both men and women.

\section{Cognitive function}

Cognitive function was assessed using the Montreal Cognitive Assessment (MoCA), which was developed to enable earlier detection of mild cognitive impairment (MCI) [29]. Since there were no consistent criteria for $\mathrm{MCI}$, the MCI diagnostic process in the current study was according guideline both from China [30] and American Academy of Neurology [31] as followed: 1) Historical report of subjective memory concerns or a change in cognition from the subject; 2) Objective evidence of impairment of cognition from MoCA; 3) Preservation of independence in functional abilities (do not have disability defined by ADL scores; 4) Not meeting the criteria for dementia by a dementia specialist. In this study, we diagnosed $\mathrm{MCI}$ based on the criteria considering both age and educational background of the studied participants in China and the cut-off of MCI among community-dwelling Chinese adults aged 80 years and over was 19 [32],

MoCA is a brief assessment of global cognitive function, visuospatial skills and executive function (0-5 scores), functions ( $0-5$ scores), delayed recall memory (0-5 scores), attention (0-6 scores), naming (0-3 scores), language (0-3 scores), abstraction (0-2 scores), and orientation. (0-6 scores) [33]. The total score of MoCA ranges from 0 to 30, with higher scores indicating better cognitive function. Besides aging, education exerts a stronger influence than MCI diagnosis on variations in MoCA scores, and this is likely to adversely affect its test performance among poorly educated individuals. In this study, we diagnosed MCI based on the criteria considering both age and educational background of the studied participants in China. According to the validated age-specific cut-points in China, participants aged 80 years and older with total MoCA score $\leq$ 19 were categorized as MCI.

\section{Covariates}

The covariates included in the analysis were sex, age, marital status (married vs. others including divorce, widowed, and never married), education, body mass index (BMI), smoke status (previous smoker vs. current smoker vs. never smoker), alcohol use, hypertension, diabetes, coronary artery disease conditions, depressive symptoms and low physical activity. Years of education was classified into three groups: below high school, high school and above high school. BMI was calculated as body weight divided by height squared and classified as underweight (<18.5), normal (18.5-23.9), overweight (24.0-27.9), and obese $(\geq 28.0)$ [34]. Physical function was assessed by HS, usual GS, time up and go test and five times sit-to-stand test. Depressive symptoms were assessed using the Korean version of 15-item geriatric depression scale (GDS-15), and participants whose scores were $\geq 8$ were suspected to have a depressive tendency. Low physical activity was assessed by the total amount of walking time for exercise purposes. Women walking less than 120 min per week and men walking less than 150 min per week were defined as low physical activity [35].

\section{Statistical analysis}

We described the socio-demographic, lifestyle, and health characteristics by AWGS-defined sarcopenia status. Normally distributed continuous variables were expressed as means \pm SDs, and non-normally distributed continuous variables were expressed as median (inter quartile range). Categorical variables were expressed as counts (percentages). Characteristics of participants according to sarcopenia status were compared using the analysis of variance test, Wilcoxon rank-sum test, chisquare test, and fisher's exact test for normally distributed continuous variables, non-normally distributed continuous variables, categorical variables, and categorical variables with small expected values, respectively. Subsequently, we used logistic regression analysis to investigate the association between cognitive impairment and sarcopenia, including its defining components. We initially adjusted for socio-demographics (age, sex, education, and marital status) and subsequently added lifestyle and health characteristics. In addition, we identified the unadjusted and adjusted linear association of each component of the sarcopenia criteria (low muscle mass, low HS, and slow GS) with different domains of cognitive function as well as global cognition, separately. The adjustment of the models followed the same aforementioned pattern. All statistical tests were two-tailed, and $P$-values $<0.05$ were considered statistically significant. All statistical analyses were performed using Stata version 14.0 (Stata, College Station, TX, USA).

\section{Results}

\section{Subjects characteristics}

Table 1 shows the characteristics of all participants according to sarcopenia status. A total of 152 (35.5\%) participants were categorized as having sarcopenia according to the 2019 AWGS criteria. Participants with sarcopenia were significantly older $(87.48 \pm 3.68$ VS $85.72 \pm 3.34, P<0.001)$, had worse nutrition assessment results $(20.39 \%$ VS $7.61 \%, P<0.001)$, higher prevalence of MCI (17.39\% VS 28.95\%, $P=0.005)$, lower HS (20.65 
Table 1 Participant's Characteristics According to Sarcopenia by 2019 AWGS criteria $(N=428)$

\begin{tabular}{|c|c|c|c|c|}
\hline & $\begin{array}{l}\text { Overall } \\
(n=428)\end{array}$ & $\begin{array}{l}\text { Without Sarcopenia } \\
(n=276)\end{array}$ & $\begin{array}{l}\text { With Sarcopenia } \\
(n=152)\end{array}$ & $P$ Value \\
\hline Age (years), mean $\pm S D$ & $86.34 \pm 3.57$ & $85.72 \pm 3.34$ & $87.48 \pm 3.68$ & $<.001$ \\
\hline Female, $n(\%)$ & $304(60.32 \%)$ & $171(61.96 \%)$ & $81(53.29 \%)$ & 0.081 \\
\hline Higher Education (above high school), n (\%) & $247(49.40 \%)$ & 139 (50.55\%) & $71(47.02 \%)$ & 0.78 \\
\hline Married, $n(\%)$ & $298(59.72 \%)$ & $164(59.42 \%)$ & $93(62.00 \%)$ & 0.603 \\
\hline BMI, $\left(\mathrm{kg} / \mathrm{m}^{2}\right)$ & $24.13 \pm 4.61$ & $25.12 \pm 4.30$ & $22.23 \pm 2.82$ & $<.001$ \\
\hline Obesity, $n(\%)$ & $108(21.43 \%)$ & $56(20.29 \%)$ & $5(3.29 \%)$ & \\
\hline Low physical activity & $240(47.62 \%)$ & $126(45.65 \%)$ & $77(50.66 \%)$ & 0.927 \\
\hline Smoke Status, $\boldsymbol{n}(\%)$ & & & & 0.79 \\
\hline Previous smoker & $8(1.60 \%)$ & $5(1.82 \%)$ & $3(1.97 \%)$ & \\
\hline Current smoker & $86(17.20 \%)$ & $45(16.42 \%)$ & $26(17.11 \%)$ & \\
\hline Never smoker & $403(80.60 \%)$ & $221(80.66 \%)$ & $123(80.92 \%)$ & \\
\hline Current Alcohol Use (>= 2-3 time/week), $n$ (\%) & $253(51.52 \%)$ & $132(48.88 \%)$ & $80(53.34 \%)$ & 0.353 \\
\hline High comorbidity (Charlsen comorbidity index $>2$ ) & $115(22.82 \%)$ & $58(21.01 \%)$ & $33(21.71 \%)$ & 0.866 \\
\hline Hypertension, $n$ (\%) & $362(73.43 \%)$ & $204(74.73 \%)$ & $104(70.75 \%)$ & 0.379 \\
\hline Diabetes mellitus, $\boldsymbol{n}(\%)$ & $137(27.57 \%)$ & $74(27.21 \%)$ & $42(27.81 \%)$ & 0.893 \\
\hline History of Coronary Artery Disease, $n$ (\%) & $264(53.88 \%)$ & $148(54.61 \%)$ & $73(49.66 \%)$ & 0.333 \\
\hline Mild Cognitive Impairment (MCl), $n$ (\%) & $152(35.51 \%)$ & $48(17.39 \%)$ & $44(28.95 \%)$ & 0.005 \\
\hline Depression, $n$ (\%) & $47(9.33 \%)$ & $23(8.33 \%)$ & $10(6.58 \%)$ & 0.646 \\
\hline Poor Self-reported Health, $n$ (\%) & $176(35.20 \%)$ & $80(28.99 \%)$ & $57(38.00 \%)$ & 0.057 \\
\hline MNA-SF $(<=11)$ & $65(12.90 \%)$ & $21(7.61 \%)$ & $31(20.39 \%)$ & $<.001$ \\
\hline \multicolumn{5}{|l|}{ Physical function } \\
\hline Handgrip Strength (kg),median (IQR) & $22.8(19.2-27.7)$ & $23.8(20.6-29.1)$ & $20.65(17.6-25.1)$ & $<.001$ \\
\hline Usual gait speed(m/s),median (IQR) & $0.85(0.67-1.02)$ & $0.93(0.73-1.06)$ & $0.79(0.64-0.94)$ & $<.001$ \\
\hline Time get up and go test (s),median (IQR) & $11.92(9.87-15.09)$ & $11.51(9.71-14.23)$ & $13.44(10.37-16.32)$ & 0.011 \\
\hline Five times Sit-to-stand(s),median (IQR) & $14(11.25-16.78)$ & $13.57(11.19-15.75)$ & $15.00(11.81-20.00)$ & 0.011 \\
\hline \multicolumn{5}{|l|}{ SMI, median (IQR) } \\
\hline Appendicular Lean Mass index $\left(\mathrm{kg} / \mathrm{m}^{2}\right)$ & $6.32(5.71-7.04)$ & $6.62(6.04-7.54)$ & $5.60(5.24-6.51)$ & 0.425 \\
\hline Appendicular Lean Mass to BMI ratio & $0.69(0.58-0.82)$ & $0.72(0.62-0.83)$ & $0.65(0.54-0.78)$ & 0.477 \\
\hline
\end{tabular}

Notes: Values are means ( \pm SD), median (inter - quartile range) or numbers (percentages). BMI body mass index, GDS Geriatric Depression Scale, MNA Mini Nutritional Assessment. $p$-values are based on the chi-square test, Fisher's exact test, and the Mann-Whitney U test. Comorbidities were hypertension, myocardial infarction, dyslipidemia, diabetes mellitus, congestive heart failure, angina pectoris, peripheral vascular disease, cerebrovascular disease, osteoarthritis, rheumarthritis, osteoporosis, asthma, or chronic obstructive pulmonary disease, as diagnosed by a physician

kg VS $23.8 \mathrm{~kg}, P<0.001)$ and lower usual GS $(0.79 \mathrm{~m} / \mathrm{s}$ VS $0.93 \mathrm{~m} / \mathrm{s}, P<0.001)$.

Table 2 shows a summary of the cognition test scores in different cognitive subdomains measured by MoCA according to sarcopenia status defined by the AWGS. The sarcopenic individuals showed significantly lower scores of the global cognitive function $(P=0.017)$ and orientation $(P=0.011)$ than non-sarcopenic individuals.

\section{Association between sarcopenia, low muscle mass, low $\mathrm{HS}$, low GS and $\mathrm{MCl}$}

The associations between sarcopenia, its defining components and risk of MCI are shown in Table 3. In the unadjusted model, sarcopenia (odds ratio $(\mathrm{OR})=1.93$,
$95 \%$ confidence interval $(\mathrm{CI})=1.21-3.09)$, low HS (OR = $2.41,95 \% \mathrm{CI}=1.57-3.69)$ and low $\mathrm{GS}(\mathrm{OR}=1.82,95 \%$ $\mathrm{CI}=1.04-3.18)$ were independently and significantly associated with higher risk of MCI. After we adjusted for age, gender, race and education, there was no significant association between low GS and MCI. After leisure-time physical activity, ADL scores, smoking status, current alcohol intake, depression, diabetes, hypertension, previous history of coronary artery disease, and obesity were further adjusted, we found that compared with participants without sarcopenia, participants with sarcopenia were associated with higher risk of MCI ( $\mathrm{OR}=1.86,95 \% \mathrm{CI}=1.04-3.33)$. Participants with low $\mathrm{HS}(\mathrm{OR}=2.33,95 \% \mathrm{CI}=1.40-3.87)$ and low $\mathrm{GS}(\mathrm{OR}=$ 
Table 2 Neuropsychological test scores according to sarcopenia by AWGS criteria

\begin{tabular}{lllll}
\hline & Overall $(\boldsymbol{n}=\mathbf{4 2 8})$ & Without Sarcopenia $(\boldsymbol{n}=\mathbf{2 7 6})$ & With Sarcopenia $(\boldsymbol{n}=\mathbf{1 5 2})$ & $\boldsymbol{P}$ Value \\
\hline MoCA total score, median (IQR) & $23(19-26)$ & $23(21-26)$ & $22(17-25)$ & $\mathbf{0 . 0 1 7}$ \\
Visuospatial \& Executive function, median (IQR) & $3(2-5)$ & $4(2-5)$ & $3(2-5)$ & $3(2-3)$ \\
Naming, median (IQR) & $3(2-3)$ & $3(2-3)$ & $5(4-6)$ & $\mathbf{0 . 0 5 6}$ \\
Attention, median (IQR) & $6(4-6)$ & $6(5-6)$ & $1(1-2)$ & 0.759 \\
Language, median (IQR) & $2(1-2)$ & $2(1-2)$ & $1(1-2)$ & 0.183 \\
Abstraction, median (IQR) & $2(1-2)$ & $2(1-2)$ & $2(0-3)$ & 0.328 \\
Delayed recall, median (IQR) & $2(0-3)$ & $2(0-4)$ & $6(5-6)$ & 0.232 \\
Orientation, median (IQR) & $6(6-6)$ & $6(6-6)$ & $\mathbf{0 . 0 6 7}$ \\
\hline
\end{tabular}

2.33, 95\% CI $=1.13-4.72$ ) were also associated with MCI. Similarly, after all potential confounders were adjusted, we found that sarcopenia $(\mathrm{OR}=3.26,95 \% \mathrm{CI}=$ 1.47-7.19), low HS (OR $=3.60,95 \% \mathrm{CI}=1.81-7.16)$ and low GS $(\mathrm{OR}=3.61,95 \% \mathrm{CI}=1.36-9.63)$ were all independent risk factors of $\mathrm{MCI}$.

\section{Association between sarcopenia, low muscle mass, low HS, low GS and performance on cognitive function}

We further analyze the association of sarcopenia and its defining components with different domains of cognition assessed by MoCA (as shown in Table 4). Sarcopenia was associated with poorer performance in global cognitive function in crude analysis, although after adjustment for sociodemographic characteristics, psychophysical confounders and lifestyle, there was no association between sarcopenia and each domain of cognition. Low HS was associated with poorer performance in global cognitive function, visuospatial and executive function, naming and delayed recall after adjusting for confounders. There were no associations between low muscle mass, low GS and domains of cognition after adjustment.

\section{Discussion}

This study demonstrated that sarcopenia and its defining component HG and GS, were significantly associated with MCI in a sample of community-dwelling oldest old in China. This study also showed that low HS was associated with performance on global and several domains of cognitive function assessed by MoCA, including visuospatial and executive function, naming and delayed recall ability.

In our study, the prevalence of sarcopenia among community-dwelling oldest old was $35.5 \%$, which was higher than people older than 60 (28.8\%) in east China [36] and people over 50 (19.31\%) in western China [37]. We found that AWGS-based sarcopenia was associated with higher risk of MCI. However, evidence from previous studies about the association between sarcopenia and cognitive function of older adults were controversial, which may be partly due to the different assessment tools of cognitive function and study population. A group study among British older men over 65 reported that no significant association was evidenced between FNIH-defined sarcopenia and mild or severe cognitive impairment assessed by MMSE [38]. Another crosssectional analysis among 3025 women aged 75 years and older also demonstrated no significant associations between different operative sarcopenia definitions and cognitive impairment (measured by short portable mental status questionnaire) [39]. By comparison, our findings were consistent with previous studies in Asian populations [4, 37, 40]. In a cross-sectional analysis of 201 community-dwelling Korean women with the mean age of 74 years old, sarcopenia was inversely associated with MMSE and CES-D scores [41]. Also, in a prospective study with 131 adults aged 65 years and older in Japan,

Table 3 Associations between sarcopenia and its defining components with risk of $\mathrm{MCl}$ in older adults $(N=396)$

\begin{tabular}{|c|c|c|c|c|c|c|c|c|}
\hline Cognitive impairment & Sarcopenia & $P$ Value & Low musclemass & $P$ Value & $\begin{array}{l}\text { Low muscle } \\
\text { strength }\end{array}$ & $P$ Value & $\begin{array}{l}\text { Low walking } \\
\text { speed }\end{array}$ & $P$ Value \\
\hline All participants & OR $(95 \% \mathrm{Cl})$ & & OR $(95 \% \mathrm{Cl})$ & & OR $(95 \% \mathrm{Cl})$ & & OR $(95 \% \mathrm{Cl})$ & \\
\hline Unadjusted Model & $1.935(1.211 \sim 3.093)$ & 0.006 & $1.471(0.943 \sim 2.295)$ & 0.089 & $2.406(1.567 \sim 3.692)$ & $<.001$ & $1.817(1.037 \sim 3.181)$ & 0.037 \\
\hline Model 1 & $1.996(1.221 \sim 3.265)$ & 0.006 & $1.482(0.933 \sim 2.354)$ & 0.095 & $2.233(1.417 \sim 3.520)$ & 0.001 & $1.736(0.981 \sim 3.071)$ & 0.058 \\
\hline Model 2 & $1.856(1.035 \sim 3.327)$ & 0.038 & $1.215(0.684 \sim 2.156)$ & 0.507 & $2.325(1.396 \sim 3.873)$ & 0.001 & $2.311(1.131 \sim 4.722)$ & 0.022 \\
\hline
\end{tabular}

Note:Model 0: unadjusted model

Model 1: logistic regression adjusted for age, sex, race, and education

Model 2: logistic regression adjusted for age, sex, race, education, leisure-time physical activity, ADL scores, smoking status, current alcohol intake, depression, diabetes, hypertension, previous history of coronary artery disease, and obesity 


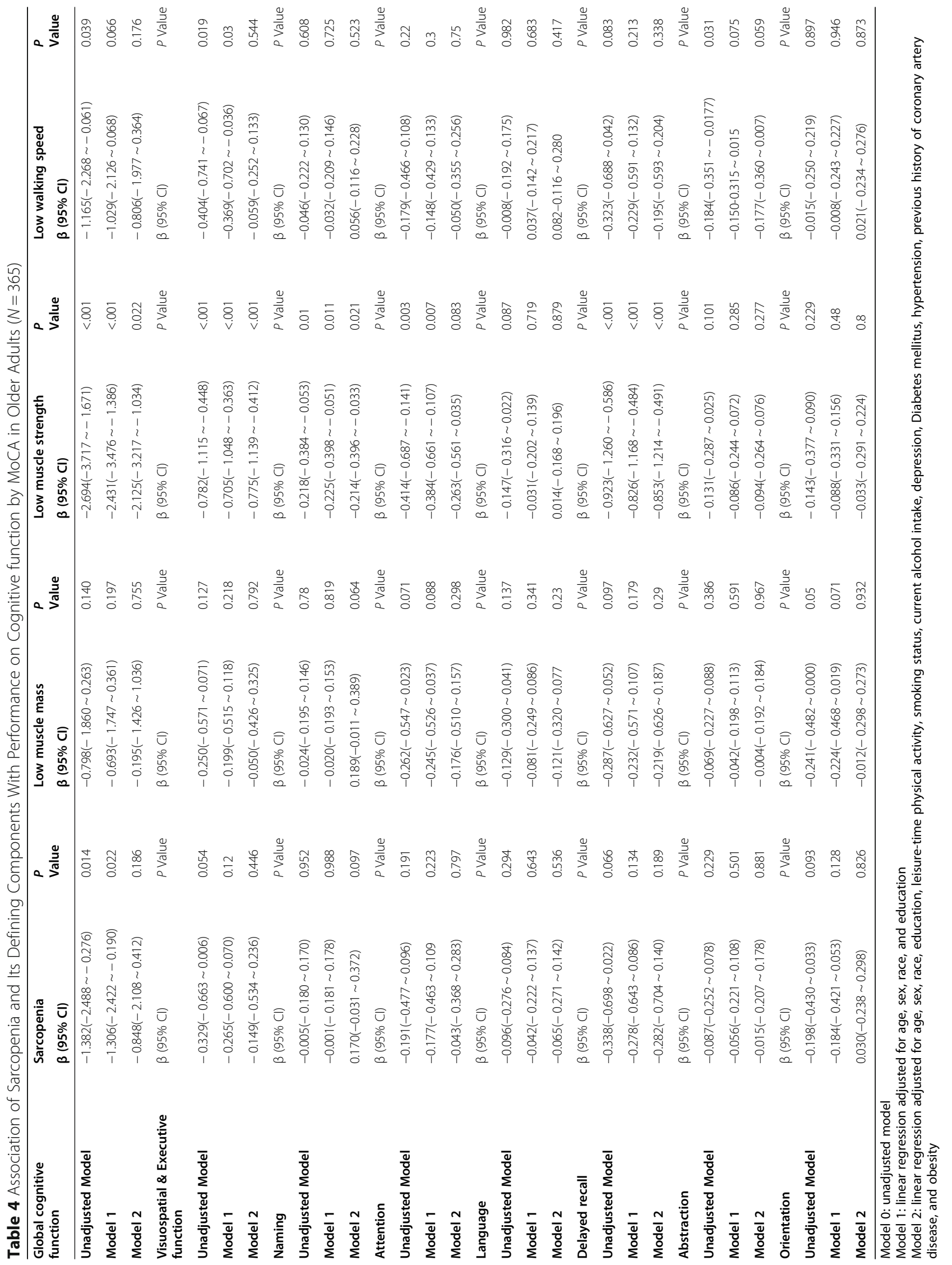


sarcopenia was founded to be an independent risk factor of cognitive deterioration assessed by MMSE during the 1 -year study period [40]. However, the aforementioned studies were not conducted among the oldest old population and failed to examine the relationship between individual defining components of sarcopenia and different domains of cognitive function. Our study used MoCA to detect the mild stages of the cognitive impairment, which was able to differentiate between distinct clinical dementia syndromes at early stages of disease with high sensitivity and specificity [42]. Additionally, in this study, we found that sarcopenia was not associated with global and different domains of cognitive function after adjustment for multiple confounder. This result was conflicting, since most of the previous studies reported that sarcopenia-related cognitive impairment was mainly in the language and executive function using MMSE and Phonemic Verbal Fluency Test. (VFT) [38, 43] The discrepancy may be due to the smaller age range of our study population. Future longitudinal studies are still needed to clarify the association between sarcopenia and cognitive subdomains among populations in this age group.

This study found that low HS was not only associated with higher risk of $\mathrm{MCI}$, but also associated with less scores in global cognitive function, visuospatial and executive function, and delayed recall in adjusted models, which indicated that low HS might be used as an early clinical screening tool for cognitive impairment among the oldest old population. Our findings are consistent with those of previous studies [11, 39, 44, 45]. Diana et.al found that poor HS is a predictor of accelerated cognitive decline using total scores of MMSE in the oldest old ( $>85$ years old) in Leiden [11]. Kim et.al also reported that baseline HS of 2378 Korean adults aged 65 or older was found to be positively associated with MMSE scores at baseline and over 8 years' time [46]. Similar to our results, one cross-sectional analysis in Brazil also showed that low muscle strength was associated with poorer performance in all assessed cognitive domains [43], while Taekema et al. only found an association with MMSE changes and not with other cognitive variables, such as memory, attention, and processing speed [47]. Whether HS could be a predictive value for cognitive decline and its exact mechanism needed to be further explored in longitudinal studies.

Also, similar to previous findings [48-51], we found that low GS was associated with MCI after adjusting for all confounders. The associations between low GS and different domains of cognitive function were not fully studied in previous studies. Our study found that GS was positively associated with global cognitive function, visuospatial and executive function and abstraction in crude analysis. However, no associations were found after full adjustment, which was inconsistent with previous studies. Annika et al. reported that low GS was associated with impairment in executive function among community-dwelling older people with CI [52]. The heterogeneity of results may be due to differences in study populations and diagnosis criteria of low GS.

The associations between low skeletal muscle mass and incidence of $\mathrm{MCI}$, as well as cognitive subdomains were also analyzed in our study. We found that low muscle mass was only associated with poorer performance in orientation in crude analysis, and no association was observed between low muscle mass and risk of MCI. Results among previous studies regarding the association between cognitive impairment and low skeletal muscle mass have been inconsistent: Won et al. found that skeletal muscle mass of elders older than 60 years old detected by bioelectrical impedance analysis (BIA) was linked with cognitive function [53], while Moon et al. showed that low muscle mass of Korean older adults was not associated with progression to cognitive impairment after 5 years of follow-up in the prospective study [54]. The cross-sectional analysis in Brazil also showed that low muscle mass was associated with poorer performance in the VFT [43] According to one systematic review, controversies among studies in the association of muscle mass with cognitive impairment were mainly due to differences of body composition devices [55], since the association might be stronger with use of BIA to measure body composition versus DEXA [56].

The possible mechanism between sarcopenia, low HS, low GS and cognitive function lies in that inflammations, oxidative stress, and hormonal changes share a common pathological role [57]. Several possible mechanisms may reinforce our conclusions that the association of sarcopenia and cognitive function is probably driven by alterations in muscle strength and not in muscle mass. First, muscle strength could reflect the change of brain-aging processes, such as the functioning of the central nervous system or white matter integrity [46]. Secondly, low HS and cognitive impairment may share common pathophysiological pathways such as systemic inflammation, insulin resistance and oxidative stress, all of which may contribute to both weak muscle strength and cognitive impairment [58]. Additionally, some studies also reported that changes in cognition and walking speed interact to predict future dementia [59]. It is thought that walking and cognition rely on similar brain regions, predominantly in the prefrontal cortex [60]. Neurodegeneration is a possible underlying mechanism linking declines in physical and cognitive function, with lower GS associated with changes in subcortical white matter and cortical gray matter volumes [61]. Another potential mechanism is neuroinflammation, which is thought to lead to impaired neuroplasticity in the brain areas controlling motor and cognitive function [60]. High 
concentrations of inflammatory markers are predictive of new-onset dementia and have also been implicated in mobility impairment [62].

The present study has many strengths. First, this is the first study to investigate the associations between sarcopenia and its defining components with different cognitive domains among Chinese community-dwelling oldest old, which provided extra support in addition to composite sarcopenia and global cognition function [63]. Our findings may have important implications in the early recognition of sarcopenia and cognitive impairment. Second, we used the MoCA scale as an early manifestation of $\mathrm{MCI}$, which was more comprehensive and sensitive than other cognition measures with good test-retest reliability [64]. Third, our participants were a reliable group of community-dwelling oldest old coming from the real world, who had electronic health records from long-run medical centers. Our interview took place in these medical centers, which guaranteed reliability and were more representative of the real world.

There are several limitations in the present study. First, we used cross-sectional models so that causality could not be proved. Longitudinal studies will be required to illustrate the relationship between sarcopenia and MCI among adults older than 80 years old. Second, this study was done using a relatively small number of cases at one retirement community. However, our data can be considered as relatively valid because it was collected using standardized methods. Third, we only used MoCA to assess cognitive performance, which has no validated subscales in several cognitive domains. Therefore, other neuropsychological testing batteries are needed to have a more comprehensive assessment of cognitive abilities in future studies.

\section{Conclusions}

The present results showed that sarcopenia was significantly associated with $\mathrm{MCI}$ among community-dwelling oldest old. Furthermore, our findings suggested that low HS may be useful to identify cognitive impairment, decreased visuospatial and executive function, naming and delayed recall at an earlier stage in non-disabled older adults living in the community. Additional longitudinal studies are needed to clarify associations between sarcopenia and its defining components and different domains of cognitive function.

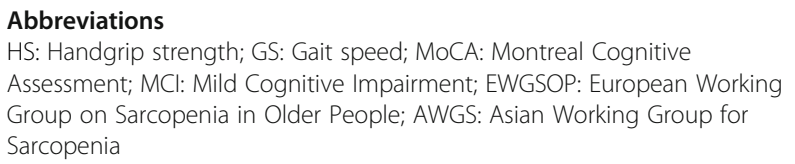

\section{Acknowledgements}

We would like to thank Ming Zhao, Shuangshuang Du, Jinxia Li, Longqin Zhang and Guangji Wang for their thoughtful contribution to this research.

\section{Authors' contributions}

Anying Bai performed the statistical analyses and drafted the manuscript. Weihao Xu collected the data. Yixin Hu contributed to the study design, collected and interpretated data, and revised the manuscript. Jing Sun, Juan Liu, Xinli Deng, Linna Wu, Xiao Zou, Jing Zuo, Lin Zou, Xunxia Liu collected the data. Hengge Xie and Xiaohong Zhang interpretated data, Li Fan revised the manuscript. All authors read and approved the final manuscript.

\section{Funding}

The study was supported by Military Healthcare Fund (15BJZ41), Military Healthcare Fund (12BJZ40) and Opening Foundation of National Clinical Research Center for Geriatric Diseases (NCRCG- PLAGH-2017010),

\section{Availability of data and materials}

The datasets used and analysed in this study are available from the corresponding author on reasonable request.

\section{Declarations}

Ethics approval and consent to participate

This study has been approved by the Research Ethics Committee of Chinese PLA General Hospital (Ethic number: S2018-102-02), and written informed consent was obtained from all participants.

\section{Consent for publication}

Not applicable.

\section{Competing interests}

The authors declare that they have no competing interests.

\section{Author details}

${ }^{1}$ Peking University Health Science Centre, School of Public Health, Beijing, China. ${ }^{2}$ Geriatric Health Care Department of The Second Medical Center \& National Clinical Research Center for Geriatric Diseases, Chinese PLA General Hospital, Beijing, China. ${ }^{3}$ Geriatric Department of Beijing North Hospital Of Ordnance Industry, Beijing, China. ${ }^{4}$ Laboratory Department of The Second Medical Center \& National Clinical Research Center for Geriatric Diseases, Chinese PLA General Hospital, Beijing, China. ${ }^{5}$ Geriatric Emergency Department of The Second Medical Center \& National Clinical Research Center for Geriatric Diseases, Chinese PLA General Hospital, Beijing, China. ${ }^{6}$ Geriatric Cardiology Department of The Second Medical Center \& National Clinical Research Center for Geriatric Diseases, Chinese PLA General Hospital, Beijing, China. ${ }^{7}$ Neurology Department of The Second Medical Center \& National Clinical Research Center for Geriatric Diseases, Chinese PLA General Hospital, Beijing, China.

Received: 27 November 2020 Accepted: 25 March 2021

Published online: 06 May 2021

\section{References}

1. Marzetti E, Calvani R, Tosato M, Cesari M, Di Bari M, Cherubini A, et al. Sarcopenia: an overview. Aging Clin Exp Res. 2017;29(1):11-7. https://doi. org/10.1007/s40520-016-0704-5.

2. Chen LK, Woo J, Assantachai P, Auyeung TW, Chou MY, lijima K, et al. Asian working Group for Sarcopenia: 2019 consensus update on sarcopenia diagnosis and treatment. J Am Med Dir Assoc. 2020;21(3):300-7.e2. https:// doi.org/10.1016/j.jamda.2019.12.012.

3. Cawthon PM, Travison TG, Manini TM, Patel S, Pencina KM, Fielding RA, et al. Establishing the link between lean mass and grip strength cut points with mobility disability and other health outcomes: proceedings of the sarcopenia definition and outcomes consortium conference. J Gerontol A Biol Sci Med Sci. 2020;75(7):1317-23. https://doi.org/10.1093/gerona/glz081.

4. Jang JY, Kim J. Association between handgrip strength and cognitive impairment in elderly Koreans: a population-based cross-sectional study. J Phys Ther Sci. 2015;27(12):3911-5. https://doi.org/10.1589/jpts.27.3911.

5. Bohannon RW. Muscle strength: clinical and prognostic value of hand-grip dynamometry. Curr Opin Clin Nutr Metab Care. 2015;18(5):465-70. https:// doi.org/10.1097/MCO.0000000000000202.

6. Arvandi M, Strasser B, Meisinger C, Volaklis K, Gothe RM, Siebert U, et al. Gender differences in the association between grip strength and mortality 
in older adults: results from the KORA-age study. BMC Geriatr. 2016;16(1): 201. https://doi.org/10.1186/s12877-016-0381-4.

7. Sobestiansky S, Michaelsson K, Cederholm T. Sarcopenia prevalence and associations with mortality and hospitalisation by various sarcopenia definitions in 85-89 year old community-dwelling men: a report from the ULSAM study. BMC Geriatr. 2019;19(1):318. https://doi.org/10.1186/s12877-01 9-1338-1.

8. Leong DP, Teo KK, Rangarajan S, Lopez-Jaramillo P, Avezum A Jr, Orlandini $A$, et al. Prognostic value of grip strength: findings from the Prospective Urban Rural Epidemiology (PURE) study. Lancet (London, Engl). 2015; 386(9990):266-73.

9. Peel NM, Alapatt LJ, Jones LV, Hubbard RE. The association between gait speed and cognitive status in community-dwelling older people: a systematic review and meta-analysis. J Gerontol A Biol Sci Med Sci. 2019; 74(6):943-8. https://doi.org/10.1093/gerona/gly140.

10. Kohara K, Okada Y, Ochi M, Ohara M, Nagai T, Tabara Y, et al. Muscle mass decline, arterial stiffness, white matter hyperintensity, and cognitive impairment: Japan Shimanami health promoting program study. J Cachexia Sarcopenia Muscle. 2017;8(4):557-66. https://doi.org/10.1002/jcsm.12195.

11. Taekema DG, Gussekloo J, Maier AB, Westendorp RG, de Craen AJ. Handgrip strength as a predictor of functional, psychological and social health. A prospective population-based study among the oldest old. Age Ageing. 2010;39(3):331-7. https://doi.org/10.1093/ageing/afq022.

12. Sternäng O, Reynolds CA, Finkel D, Ernsth-Bravell M, Pedersen NL, Dahl Aslan AK. Grip strength and cognitive abilities: associations in old age. J Gerontol Ser B Psychol Sci Soc Sci. 2016;71(5):841-8. https://doi.org/10.1093/geronb/gbv017.

13. Hsu CL, Liang CK, Liao MC, Chou MY, Lin YT. Slow gait speed as a predictor of 1-year cognitive decline in a veterans' retirement community in southern Taiwan. Geriatr Gerontol Int. 2017;17(Suppl 1):14-9. https://doi.org/10.1111/ ggi.13034.

14. Grande G, Triolo F, Nuara A, Welmer AK, Fratiglioni L, Vetrano DL. Measuring gait speed to better identify prodromal dementia. Exp Gerontol. 2019;124: 110625. https://doi.org/10.1016/j.exger.2019.05.014.

15. McGrath R, Vincent BM, Hackney KJ, Robinson-Lane SG, Downer B, Clark BC. The Longitudinal Associations of Handgrip Strength and Cognitive Function in Aging Americans. J Am Med Dir Assoc. 2020;21(5):634-9 e631.

16. Callisaya ML, Blizzard CL, Wood AG, Thrift AG, Wardill T, Srikanth VK. Longitudinal relationships between cognitive decline and gait slowing: the Tasmanian study of cognition and gait. J Gerontol A Biol Sci Med Sci. 2015; 70(10):1226-32. https://doi.org/10.1093/gerona/glv066.

17. Muir SW, Speechley M, Wells J, Borrie M, Gopaul K, Montero-Odasso M. Gait assessment in mild cognitive impairment and Alzheimer's disease: the effect of dual-task challenges across the cognitive spectrum. Gait Posture. 2012; 35(1):96-100. https://doi.org/10.1016/.jgaitpost.2011.08.014.

18. van Kan GA, Cesari M, Gillette-Guyonnet S, Dupuy C, Vellas B, Rolland Y. Association of a 7-year percent change in fat mass and muscle mass with subsequent cognitive dysfunction: the EPIDOS-Toulouse cohort. J Cachexia Sarcopenia Muscle. 2013;4(3):225-9.

19. Xu X, Chong E, Hilal S, Ikram MK, Venketasubramanian N, Chen C. Beyond Screening: Can the Mini-Mental State Examination be Used as an Exclusion Tool in a Memory Clinic? Diagnostics (Basel, Switzerland). 2015;5(4):475-86.

20. Spering CC, Hobson V, Lucas JA, Menon CV, Hall JR, O'Bryant SE. Diagnostic accuracy of the MMSE in detecting probable and possible Alzheimer's disease in ethnically diverse highly educated individuals: an analysis of the NACC database. J Gerontol Series A. 2012;67(8):890-6. https://doi.org/10.1 093/gerona/gls006.

21. Kazazi L, Foroughan M, Nejati V, Shati M. Association between age associated cognitive decline and health related quality of life among Iranian older individuals. Electron Physician. 2018;10(4):6663-71. https://doi.org/10.1 9082/6663.

22. Tao J, Jin YF, Yang Z, Wang LC, Gao XR, Lui L, et al. Reduced arterial elasticity is associated with endothelial dysfunction in persons of advancing age: comparative study of noninvasive pulse wave analysis and laser Doppler blood flow measurement. Am J Hypertens. 2004;17(8):654-9. https://doi.org/10.1016/j.amjhyper.2004.03.678.

23. Gillis C, Mirzaei F, Potashman M, Ikram MA, Maserejian N. The incidence of mild cognitive impairment: A systematic review and data synthesis. Alzheimer's Dementia (Amsterdam, Netherlands). 2019;11:248-56.

24. Tang $Y$. The MoCA as a cognitive screening tool for mild cognitive impairment (MCI) in elderly adults in China. Psychiatry Res. 2020;291:113210. https://doi.org/10.1016/j.psychres.2020.113210.
25. Wallin A, Nordlund A, Jonsson M, Lind K, Edman $\AA$, Göthlin $M$, et al. The Gothenburg MCl study: design and distribution of Alzheimer's disease and subcortical vascular disease diagnoses from baseline to 6-year follow-up. J Cereb Blood Flow Metab. 2016;36(1):114-31. https://doi.org/10.1038/ jcbfm.2015.147.

26. Zeng Y, Feng Q, Hesketh T, Christensen K, Vaupel JW. Survival, disabilities in activities of daily living, and physical and cognitive functioning among the oldest-old in China: a cohort study. Lancet (London, England). 2017; 389(10079):1619-29.

27. Taekema DG, Maier AB, Westendorp RG, de Craen AJ. Higher blood pressure is associated with higher handgrip strength in the oldest old. Am J Hypertens. 2011;24(1):83-9. https://doi.org/10.1038/ajh.2010.185.

28. Hu X, Zeng Y, Zhen X, Zhang H, Li Y, Gu S, et al. Cognitive and physical function of people older than 80 years in China from 1998 to 2014. J Int Med Res. 2018;46(7):2810-27. https://doi.org/10.1177/0300060518773211.

29. Li X, Jia S, Zhou Z, Jin Y, Zhang X, Hou C, et al. The role of the Montreal cognitive assessment (MoCA) and its memory tasks for detecting mild cognitive impairment. Neurol Sci. 2018;39(6):1029-34. https://doi.org/10.1 007/s10072-018-3319-0.

30. Du YF, Lv PY, Ji Y, Wu LY, Shi L. 2018 Chinese guidelines for the diagnosis and treatment of dementia and cognitive impairment (5): diagnosis and treatment of mild cognitive impairment. Chin Med J. 2018;(17):1294-301. doi:CNKI:SUN:ZHYX.0.2018-17-004.

31. Petersen RC, Lopez O, Armstrong MJ, Getchius TSD, Ganguli M, Gloss D, et al. Practice guideline update summary: mild cognitive impairment: report of the guideline development, dissemination, and implementation Subcommittee of the American Academy of neurology. Neurology. 2018;90(3):126-35.

32. Meng XM, Yu FC, Yin J, Tang XM, Chen XP, Liu YZ, et al. A preliminary study of Montreal cognitive assessment scale in patients with mild cognitive impairment. Chin J Geriatr Cardio Cerebrovasc Dis. 2012;14(11):1173-5.

33. Trzepacz PT, Hochstetler H, Wang S, Walker B, Saykin AJ. Alzheimer's disease neuroimaging I: relationship between the Montreal cognitive assessment and mini-mental state examination for assessment of mild cognitive impairment in older adults. BMC Geriatr. 2015;15(1):107. https://doi.org/10.11 86/s12877-015-0103-3.

34. Ali A, Rahut DB. Healthy foods as proxy for functional foods: Consumers' awareness, perception, and demand for natural functional foods in Pakistan. Int J Food Sci. 2019:6390650

35. Jia RX, Liang JH, Xu Y, Wang YQ. Effects of physical activity and exercise on the cognitive function of patients with Alzheimer disease: a meta-analysis. BMC Geriatr. 2019;19(1):181. https://doi.org/10.1186/s12877-019-1175-2.

36. Yang $L$, , Wu GH, Yang $Y L$, Wu YH, Zhang L, Wang MH, et al. Nutrition, physical exercise, and the prevalence of sarcopenia in elderly residents in nursing homes in China. Med Sci Monitor. 2019;25:4390-9. https://doi.org/1 0.12659/MSM.914031.

37. Liu X, Hou L, Xia X, Liu Y, Zuo Z, Zhang Y, et al. Prevalence of sarcopenia in multi ethnics adults and the association with cognitive impairment: findings from West-China health and aging trend study. BMC Geriatr. 2020;20(1):63. https://doi.org/10.1186/s12877-020-1468-5.

38. Huang C-Y, Hwang A-C, Liu L-K, Lee W-J, Chen L-Y, Peng L-N, et al. Association of Dynapenia, sarcopenia, and cognitive impairment among community-dwelling older Taiwanese. Rejuvenation Res. 2016;19(1):71-8. https://doi.org/10.1089/rej.2015.1710.

39. Abellan van Kan G, Cesari M, Gillette-Guyonnet S, Dupuy C, Nourhashémi F, Schott AM, et al. Sarcopenia and cognitive impairment in elderly women: results from the EPIDOS cohort. Age Ageing. 2013;42(2):196-202.

40. Nishiguchi S, Yamada M, Shirooka H, Nozaki Y, Fukutani N, Tashiro Y, et al. Sarcopenia as a Risk Factor for Cognitive Deterioration in CommunityDwelling Older Adults: A 1-Year Prospective Study. J Am Med Dir Assoc. 2016;17(4):372 e375-378.

41. Lee I, Cho J, Hong H, Jin Y, Kim D, Kang H. Sarcopenia is associated with cognitive impairment and depression in elderly Korean women. Iran J Public Health. 2018:47(3):327-34.

42. Wood JL, Weintraub S, Coventry C, Xu J, Zhang H, Rogalski E, et al. Montreal cognitive assessment (MoCA) performance and domainspecific index scores in amnestic versus aphasic dementia. J Int Neuropsychol Soc. 2020:1-5.

43. Szlejf C, Suemoto CK, Lotufo PA, Benseñor IM, Newman A. Association of sarcopenia with performance on multiple cognitive domains: results from the ELSA-Brasil study. J Gerontol: Series A. 2019;74(11):1805-11. https://doi. org/10.1093/gerona/glz118. 
44. Auyeung TW, Lee JS, Kwok T, Woo J. Physical frailty predicts future cognitive decline - a four-year prospective study in 2737 cognitively normal older adults. J Nutr Health Aging. 2011;15(8):690-4. https://doi.org/10.1007/s12 603-011-0110-9.

45. Auyeung TW, Kwok T, Lee J, Leung PC, Leung J, Woo J. Functional decline in cognitive impairment--the relationship between physical and cognitive function. Neuroepidemiology. 2008;31(3):167-73. https://doi.org/10.1159/ 000154929.

46. Kim KH, Park SK, Lee DR, Lee J. The relationship between handgrip strength and cognitive function in elderly Koreans over 8 years: a prospective population-based study using Korean longitudinal study of ageing. Korean J Fam Med. 2019;40(1):9-15. https://doi.org/10.4082/ kjfm.17.0074.

47. Taekema DG, Ling CH, Kurrle SE, Cameron ID, Meskers CG, Blauw GJ, et al. Temporal relationship between handgrip strength and cognitive performance in oldest old people. Age Ageing. 2012;41(4):506-12. https:// doi.org/10.1093/ageing/afs013.

48. Quan M, Xun P, Chen C, Wen J, Wang Y, Wang R, et al. Walking pace and the risk of cognitive decline and dementia in elderly populations: a metaanalysis of prospective cohort studies. J Gerontol A Biol Sci Med Sci. 2017; 72(2):266-70. https://doi.org/10.1093/gerona/glw121.

49. Demnitz $N$, Esser $P$, Dawes $H$, Valkanova V, Johansen-Berg $H$, Ebmeier $\mathrm{KP}$, et al. A systematic review and meta-analysis of cross-sectional studies examining the relationship between mobility and cognition in healthy older adults. Gait Posture. 2016;50:164-74. https://doi.org/10.101 6/j.gaitpost.2016.08.028.

50. Kueper JK, Speechley M, Lingum NR, Montero-Odasso M. Motor function and incident dementia: a systematic review and meta-analysis. Age Ageing. 2017;46(5):729-38. https://doi.org/10.1093/ageing/afx084.

51. Beauchet $\mathrm{O}$, Annweiler C, Callisaya ML, De Cock AM, Helbostad JL, Kressig RW, et al. Poor gait performance and prediction of dementia: results from a meta-analysis. J Am Med Dir Assoc. 2016;17(6):482-90. https://doi.org/10.101 6/j.jamda.2015.12.092.

52. Toots ATM, Taylor ME, Lord SR, Close JCT. Associations between gait speed and cognitive domains in older people with cognitive impairment. J Alzheimer's Dis. 2019;71(s1):S15-s21. https://doi.org/10.3233/JAD-181173.

53. Won H, Abdul Manaf Z, Mat Ludin AF, Shahar S. Wide range of body composition measures are associated with cognitive function in community-dwelling older adults. Geriatr Gerontol Int. 2017;17(4):554-60. https://doi.org/10.1111/ggi.12753.

54. Moon JH, Moon JH, Kim KM, Choi SH, Lim S, Park KS, et al. Sarcopenia as a predictor of future cognitive impairment in older adults. J Nutr Health Aging. 2016;20(5):496-502. https://doi.org/10.1007/s12603-015-0613-x.

55. Chang KV, Hsu TH, Wu WT, Huang KC, Han DS: Association Between Sarcopenia and Cognitive Impairment: A Systematic Review and MetaAnalysis. J Am Med Dir Assoc 2016, 17; (12): 1164.e1167-1164.e1115.

56. Kim M, Won CW. Sarcopenia Is Associated with Cognitive Impairment Mainly Due to Slow Gait Speed: Results from the Korean Frailty and Aging Cohort Study (KFACS). Int J Environ Res Public Health. 2019;16(9):1491. https://doi.org/10.3390/ijerph16091491.

57. Namioka N, Hanyu H, Hirose D, Hatanaka H, Sato T, Shimizu S. Oxidative stress and inflammation are associated with physical frailty in patients with Alzheimer's disease. Geriatr Gerontol Int. 2017;17(6):913-8. https://doi.org/1 $0.1111 /$ ggi.12804.

58. Mangialasche F, Polidori MC, Monastero R, Ercolani S, Camarda C, Cecchetti $\mathrm{R}$, et al. Biomarkers of oxidative and nitrosative damage in Alzheimer's disease and mild cognitive impairment. Ageing Res Rev. 2009;8(4):285-305. https://doi.org/10.1016/j.arr.2009.04.002.

59. Hackett RA, Davies-Kershaw H, Cadar D, Orrell M, Steptoe A. Walking speed, cognitive function, and dementia risk in the English longitudinal study of ageing. J Am Geriatr Soc. 2018;66(9):1670-5. https://doi.org/1 $0.1111 /$ jgs. 15312 .

60. Valkanova V, Ebmeier KP. What can gait tell us about dementia? Review of epidemiological and neuropsychological evidence. Gait Posture. 2017:53: 215-23. https://doi.org/10.1016/j.gaitpost.2017.01.024.

61. Holtzer R, Epstein N, Mahoney JR, Izzetoglu M, Blumen HM. Neuroimaging of mobility in aging: a targeted review. J Gerontol A Biol Sci Med Sci. 2014; 69(11):1375-88. https://doi.org/10.1093/gerona/glu052.

62. Sorond FA, Cruz-Almeida Y, Clark DJ, Viswanathan A, Scherzer CR, De Jager $P$, et al. Aging, the central nervous system, and mobility in older adults: neural mechanisms of mobility impairment. J Gerontol A Biol Sci Med Sci. 2015;70(12):1526-32. https://doi.org/10.1093/gerona/glv130.

63. Peng TC, Chen WL, Wu LW, Chang YW, Kao TW. Sarcopenia and cognitive impairment: a systematic review and meta-analysis. Clin Nutr. 2020;39(9): 2695-701. https://doi.org/10.1016/j.clnu.2019.12.014. Epub 2019 Dec 17.

64. Chen LK, Hwang AC, Lee WJ, Peng LN, Lin MH, Neil DL, Shih SF, Loh CH, Chiou ST. Efficacy of multidomain interventions to improve physical frailty, depression and cognition: data from cluster-randomized controlled trials. J Cachexia Sarcopenia Muscle. 2020;11(3):650-62. https://doi.org/10.1002/ jcsm.12534. Epub 2020 Mar 5.

\section{Publisher's Note}

Springer Nature remains neutral with regard to jurisdictional claims in published maps and institutional affiliations.
Ready to submit your research? Choose BMC and benefit from:

- fast, convenient online submission

- thorough peer review by experienced researchers in your field

- rapid publication on acceptance

- support for research data, including large and complex data types

- gold Open Access which fosters wider collaboration and increased citations

- maximum visibility for your research: over $100 \mathrm{M}$ website views per year

At BMC, research is always in progress.

Learn more biomedcentral.com/submissions 